\title{
Homogeneous and heterogeneous propagation of COVID-19 from super-spreading to super-isolation
}

\section{Jack Sutton}

Nottingham Trent University

Golnaz Shahtahmassebi

Nottingham Trent University

Haroldo V. Ribeiro

State University of Maringa

Quentin S. Hanley ( $\nabla$ quentin.hanley@ntu.ac.uk)

Nottingham Trent University

\section{Research Article}

Keywords: COVID-19, Density Scaling Metrics, Rural Scaling, Urban Scaling, Population Density, Distance

Posted Date: March 11th, 2021

DOl: https://doi.org/10.21203/rs.3.rs-276177/v1

License: (c) (i) This work is licensed under a Creative Commons Attribution 4.0 International License. Read Full License 


\section{Homogeneous and heterogeneous propagation of COVID-19 from super-spreading to super-isolation}

Jack Sutton, ${ }^{*}$ Golnaz Shahtahmassebi, ${ }^{*}$ Haroldo V. Ribeiro, ${ }^{*}$ and Quentin S.

Hanley $^{*}$

*School of Science and Technology

Nottingham Trent University

Nottingham NG11 8NS

United Kingdom

fDepartamento de Física,

Universidade Estadual de Maringá,

Maringá, PR 87020-900,

Brazil

Corresponding author: Q.S. Hanley (quentin.hanley@ntu.ac.uk)

February 2021

Key words: COVID-19, Density Scaling Metrics, Rural Scaling, Urban Scaling, Population

Density, Distance 


\begin{abstract}
:
We investigated daily COVID-19 cases and death in the 337 lower tier local authority regions in England and Wales to better understand how the disease propagated over a 10-month period. Population density scaling models revealed residual variance and skewness to be sensitive indicators of the dynamics of propagation. Lockdowns and schools reopening triggered increased variance indicative of outbreaks with local impact and country scale heterogeneity. University reopening and December holidays triggered reduced variance indicative of country scale homogenisation which reached a minimum after New Year. Homogeneous propagation was associated with better correspondence with normally distributed residuals while heterogeneous propagation was more consistent with skewed models. Skewness varied from strongly negative to strongly positive revealing an unappreciated feature of community propagation. Hot spots and super-spreading events are well understood descriptors of regional disease dynamics that would be expected to be associated with positively skewed distributions. Positively skewed behaviour was observed; however, negative skewness indicative of "coldspots" and "super-isolation" dominated for approximately 4 months during the period of study. In contrast, death metrics showed near constant behaviour in scaling, variance, and skewness metrics over the full period with rural regions preferentially affected, an observation consistent with regional age demographics in England and Wales.
\end{abstract}




\section{Introduction:}

SARS-CoV-2 spread rapidly from a cluster of cases in China in December 2019 to a global pandemic on 13 March 2020. The number of confirmed cases of COVID-19 continues to grow worldwide with over 112 million cases and nearly 2.5 million deaths. SARS-CoV-2 is thought to spread by direct contact, fomites, and aerosols from both symptomatic and asymptomatic people [1-4]. During the first year of the pandemic, distancing measures and meeting size restrictions have been widely deployed to slow the spread of the disease by reducing the number and duration of interactions capable of causing infection. At scale, population density could be a proxy for these interactions. For example, someone living in a region of high population density is expected to have a greater number of interactions compared with someone who lives in a rural setting [5].

The effects of population size on COVID-19 dynamics have been investigated previously including aspects of population density effects [6-9]. Investigations of population density effects have been limited to a relatively small number of time points aggregated over a period of time, usually a month or year [10-16]. Daily granularity of data is not easily accessible; however, the COVID-19 pandemic has provided a unique and evolving data set with daily updates. These data have been influential in informing government interventions, policy decisions, and public perceptions allowing data driven informed decisions [17].

These daily data at relatively high regional granularity provide an opportunity to document the daily evolution of scaling metrics and residual variance over an extended period. Here, we investigated scaling behaviour in England and Wales using daily COVID-19 cases and death in England and Wales Lower Tier Local Authorities (LTLAs) with population density. These were examined to better understand how infectious disease metrics progress over time at country scale. 


\section{Theory:}

Scaling Models. Urban scaling [18] considers population to predict a range of urban indicators. A variety of mathematical forms have been applied with power laws being widely used.

\begin{tabular}{|r|l|}
\hline$Y \sim P^{\beta}$ & $(1)$ \\
\hline
\end{tabular}

Here, $Y$ is the indicator, $P$ is the population and $\beta$ is the scaling exponent. An estimate to the parameter $\beta$, can be obtained by applying the least square method to the logarithmic version of equation 1 (i.e. $\log y v s . \log P$ ).

When combining rural and urban regions, density metrics provide better models [12-13] than population. This can be described by similar power-law functions of the form

$$
Y / A \sim(P / A)^{\beta_{D}}
$$

Similarly to population scaling, when $\beta_{D}<1$ scaling is sub-linear, when $\beta_{D}=1$, the scaling is linear and when $\beta_{D}>1$ the scaling is super-linear. When interpreting density scaling results, sub-linear scaling accelerates in rural (low-density) regions and super-linear scaling accelerates in urban (high density) areas. The log transformed data is usually fitted to the logarithmic form

$$
\log (Y / A)=\log y_{o}+\beta_{D} \log (P / A)
$$

to obtain the parameters.

The residuals, $z_{i}$, from the fits to the models defined in equations 2-4 using method of least squares provide density scale adjusted metrics (DSAMs). If DSAMs are negative, then this is below the expectation and if DSAMs are positive then this is above expectation. 
Residual and Case Density Models. The distribution of residuals obtained from the England was modelled using normal and generalised logistic (GL) distributions. The latter has the form,

\begin{tabular}{|l|l|}
\hline$G L(x ; \theta, \sigma, \alpha)=\frac{\alpha}{\sigma} \frac{e^{-\frac{x-\theta}{\sigma}}}{\left\{1+e^{-\frac{x-\theta}{\sigma}}\right\}^{\alpha+1}}$ & \\
\hline
\end{tabular}

where $\theta, \sigma$ and $\alpha$ are the location, scale and shape parameters respectively such that $\alpha>0$, $\sigma>0$ and $-\infty<x<+\infty$. The first moment of the GL is $E(X)=\theta+\sigma(\Psi(\alpha)-\Psi(1))$ where $\Psi(1) \cong-0.57721$ and $\psi$ the digamma function. The second moment of the GL distribution is $\operatorname{Var}(X)=\sigma^{2}\left(\frac{\pi^{2}}{6}+\Psi^{\prime}(\alpha)\right)$.

\section{Results and Discussion:}

Overview of regions, cases, and number of observations. England and Wales have 337 LTLAs which range in area from 1213 ha (Kensington and Chelsea) up to 518,037 ha (Powys) and have populations between 37,340 (Rutland) up to 1,070,912 (Birmingham). Population densities vary from 0.25 people per hectare (p/ha) (Eden) to $138 \mathrm{p} / \mathrm{ha}$ (Islington). Not all LTLAs reported cases or deaths on each day within the period leading to variability in observations (Figure 1). This largely tracked the general progress of the pandemic with the summer months showing the fewest cases, deaths and observations. Histograms of per capita cases (Fig. 2) exhibited variable shapes over the course of the pandemic with some periods showing negative skew (Fig. 2(a)) while at others they were positively skewed (Fig. 2(b)). The availability of testing varied widely over the 10 months which may be a confounder in some presentations; however, the daily scaling metrics, variance, and skewness will reflect the processes in place on the day and were not obviously aligned with testing or the number of observations. All daily per capita case histograms can be found in Figure S1 in the supplementary material. 


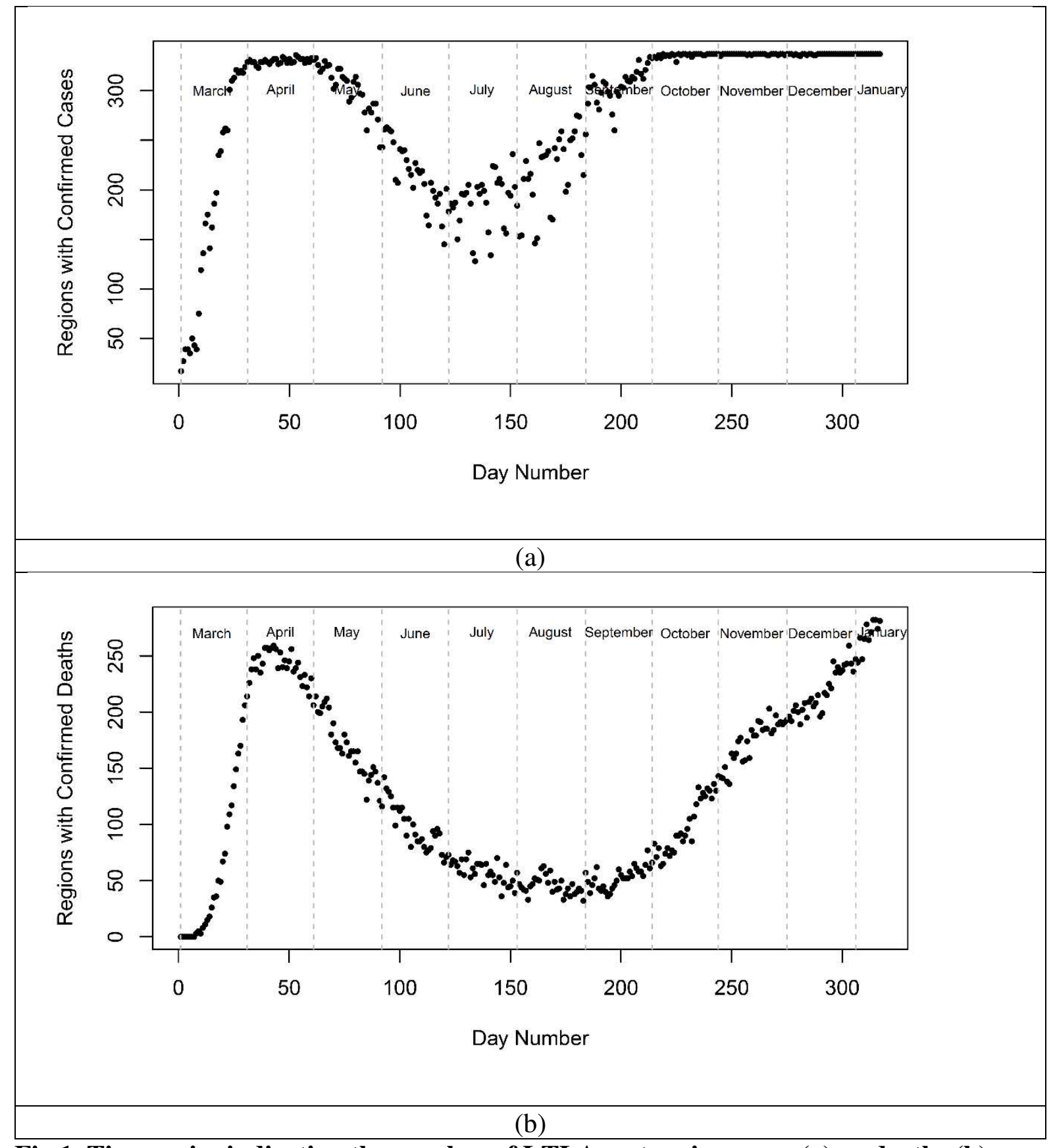

Fig 1. Time series indicating the number of LTLAs returning cases (a) or deaths (b) over the period of study. 


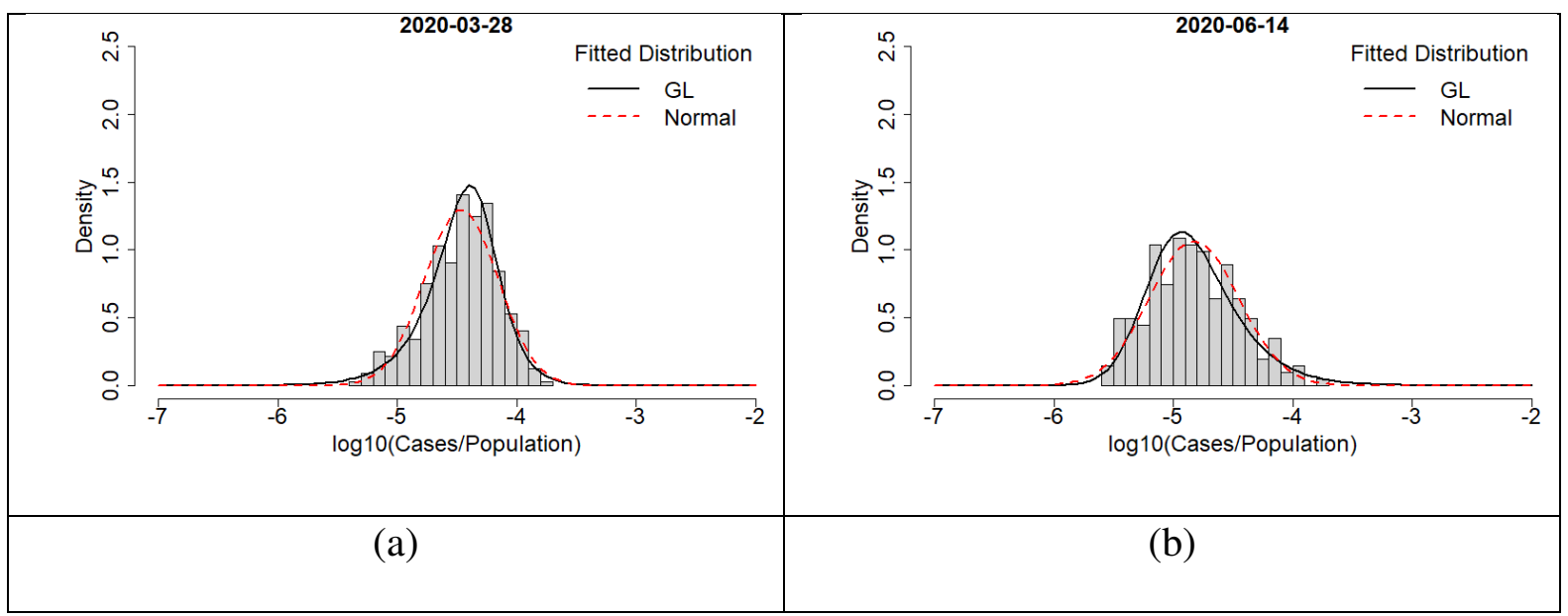

Fig 2. Histograms of per capita cases in English and Welsh LTLAs. Some periods within the time series showed negative skew (a) while others were positively skewed (b).

Daily Progression of COVID-19. To test for scaling behaviour and to correct for the known bias of per capita measures daily scaling plots (Figure 3 (a), (b), (c) and (d): and Figures S2 and S3) were constructed and found to be consistent with single power-law models throughout the pandemic. The daily residuals obtained were used as scale adjusted metrics to create geomaps (Figure 3 (e), (f), (g) and (h): Figures S4 and S5). Overall, regions displayed in the daily geomaps were relatively consistent in their position relative to the scaling law over time despite the rise and fall of daily cases (Figure S6). The persistence of regional positions relative to the scaling laws indicates that the notion of local "surges" of COVID-19 may be less important than persistent local features after the first few weeks of the pandemic. DSAMs are more useful metrics that could be used to assist local interventions.

In the scaling plots (Fig. 3 (a-d)), variability in residual variance was clear by inspection. For example, toward the end of the December holiday period (31/12/2020; Fig. 3(d)) the data were closer to the power law than in September (30/9/2020; fig 3(c)). The low variance periods represent a more homogenous presentation of cases across the regions while the higher variance periods were indicative of more heterogeneous regional cases. All daily scaling plots and corresponding geomaps can be found in Figures S2, S3, S4 and S5 in the supplementary material. 


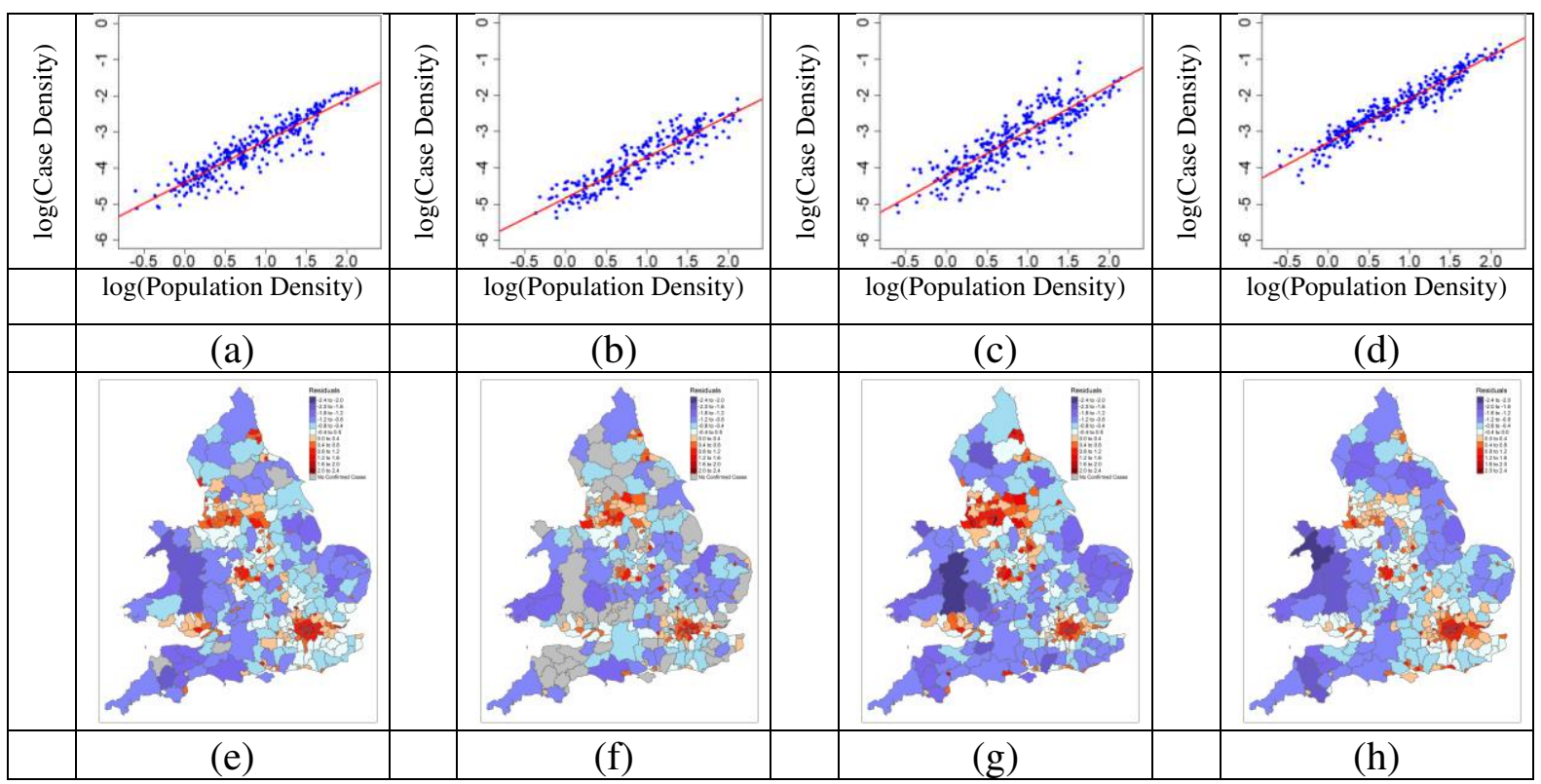

Fig 3. Scaling plots and geoplots at different times during the pandemic. These are recorded on the (a and e) 31/03/2020. (b and f) 31/8/2020. (c and g) 30/09/2020. (d and h) $31 / 12 / 2020$. Regions that are red are above expectation and blue is below. The darker the shade the further from the scaling law.

Daily Exponent, Variance, and Skewness for Cases. The LTLA data were examined to assess the trajectory of scaling behaviour, residual variance, and skewness over 10 months of the pandemic for cases (Fig 4). The scaling exponents (Fig. 4(a)) for cases rose quickly reaching a peak near the beginning of the first lockdown (announced on the 23/03/2020) in the England and Wales and declined gradually until restrictions were slowly eased toward the end of May and early June. The peak in cases during the first three months coincides with super-linear scaling; however, super-linear scaling was not universal and the preference for propagation in rural vs. urban regions reversed three times during the period of study: early-March, late April, and the end of July. Although periods of rapid growth, seem to coincide with acceleration in high population density regions the long-term behaviour of the pandemic makes clear there is no universal rule and population density is not a simple proxy for infectious interactions. 


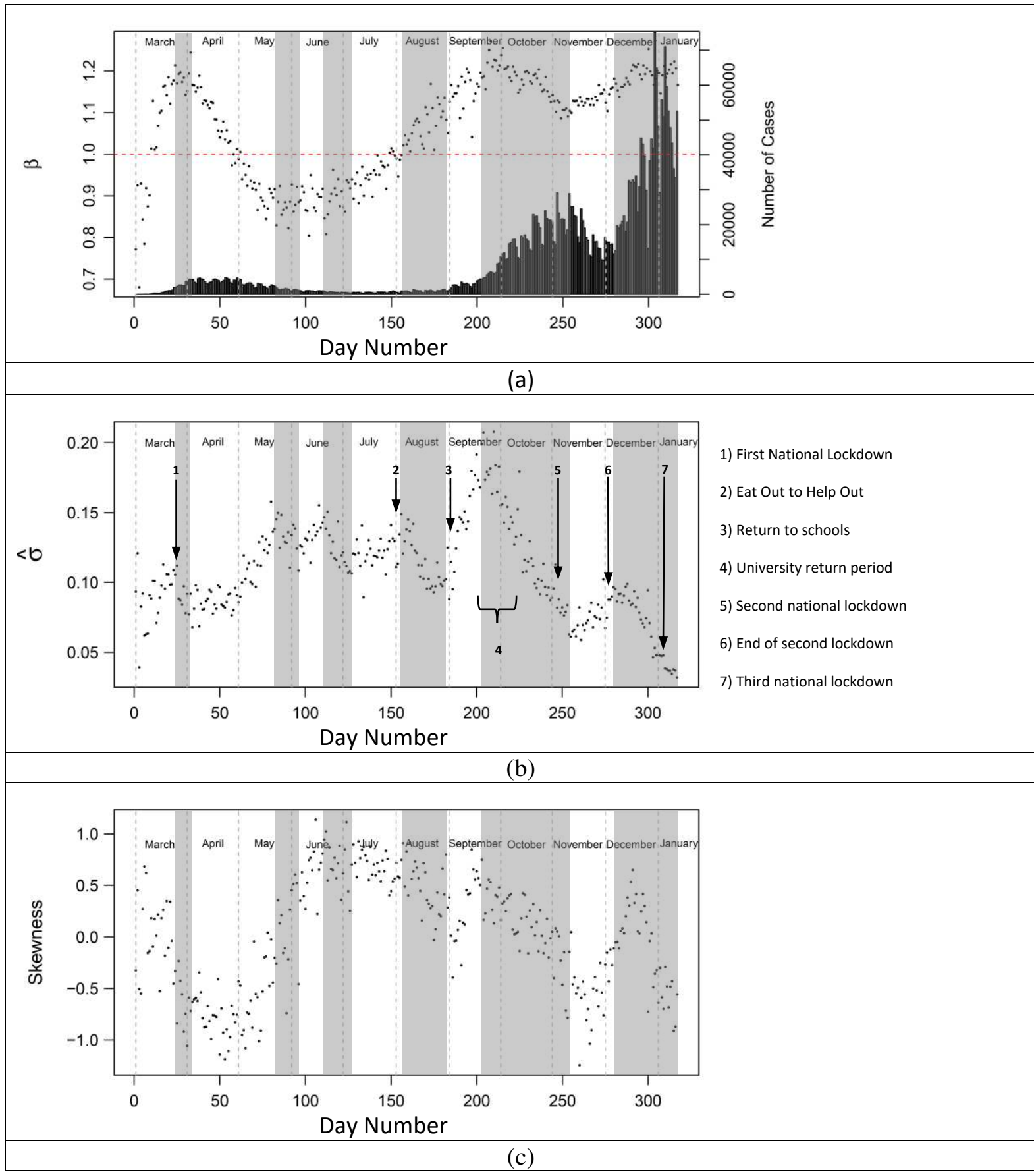

Fig 4. Daily time series of scaling exponent and residual variance and skewness for cases between 01/03/2020 and 11/01/2021. (a) Time series of daily scaling exponent of COVID-19 cases, (b) residual variance, and (c) residual skewness. The horizontal line in (a) indicates linear scaling. The bar chart indicates raw daily cases. The grey shading indicates periods of homogenisation. Arrows indicate key dates/time periods. The second lockdown in Wales preceded England beginning on 20/10/2020.

The trajectory of residual variance (Fig 4(b)) changed by over a factor of 4 during the 10 month period and presented a contrast to the scaling parameters. Variance remained relatively constant 
until late April and the later stages of the first lockdown when regional heterogeneity increased distances from the scaling law. Released restrictions tended to homogenize and reduce the distance of individual regions to the scaling law. In the summer months, regional lockdowns (Leicester and greater Manchester) in late June were followed by increasing variance. Residual variance grew rapidly following the opening of schools around (First week of September), doubling in approximately 2 weeks suggesting that schools assisted in propagating cases, but the impact was mostly contained within their immediate regions. Although an immediate "surge" in cases was not seen, a continuation of a consistent increase in cases that began in August was observed. The increasing variance indicates heterogeneous propagation that continued until mid-September when the trend reversed until the beginning of the second national lockdown in November (05/11/2020).

The period of declining variance and homogenisation coincides with students returning to Universities. There are approximately 2.4 million students attending universities in the UK. University teaching terms have staggered start dates from the last weeks of September through the first weeks in October. These typically follow a week of orientation and social activities. In advance of orientation and the start of teaching, many students travel with their families from all parts of the UK along with a large number of students who arrive from abroad. This process changed the dynamics of propagation in England and Wales during this time. While there may be other explanations in addition to Universities re-opening, there are no other obvious country scale policy changes or processes during this time window.

Homogenisation also occurred following the release of the second lockdown (03/12/2020) through to the end of the period of study. Notably, only the abrupt release of the second lockdown is associated with an obvious "surge" in cases. This includes the major holidays of Christmas and New Year's. Neither caused a "surge." They continued the propagation of the disease in a way that was consistent before and after these key dates. The general country scale 
homogenisation between the LTLA regions drove residual variance to the lowest levels seen over the 10-month period.

Skewness provides a further contrast to case counts, scaling exponents, and variance. We used the scaling law residuals to create a time series of skewness metrics (Fig. 4(c)). Similar behaviour was seen in the per capita case distributions (Fig. 2) with characteristics changing over the course of the 10-month period. When cases follow a distribution with a strong positive skew, the long positive tail of the skewed distribution is indicative of propagation with hot spots and potential super-spreading incidents. Conversely, when the residuals are negatively skewed, this indicates a distribution better characterised by a long tail of "cold spots" or superisolated regions.

\section{Daily Exponent, Variance, and Skewness for Deaths.}

In contrast, daily exponents, variance, and skewness for COVID-19 death (Fig. 5) were consistent and remained at a similar level throughout the pandemic. For a short time at the beginning of the time series, regions exhibited super-linear scaling, but this inverted circa 10/04/2020 and remained sub-linear thereafter. This meant that for most of the pandemic rural regions were preferentially affected by death. Variance and skewness also do not change much and have very little structure in comparison to cases. This shows constant homogeneous behaviour and similarly distributed death throughout England and Wales. This is in stark contrast with the far greater structure in cases. This behaviour is consistent with the age demographics in England and Wales. Previous work has documented that populations dense regions serve and a magnet for young people while rural regions tend to have a greater proportion of elderly people [11]. The scaling exponents for death throughout are consistent with those seen for scaling of people 60 and above in England and Wales. This is overwhelmingly the demographic most likely to die from COVID-19. 


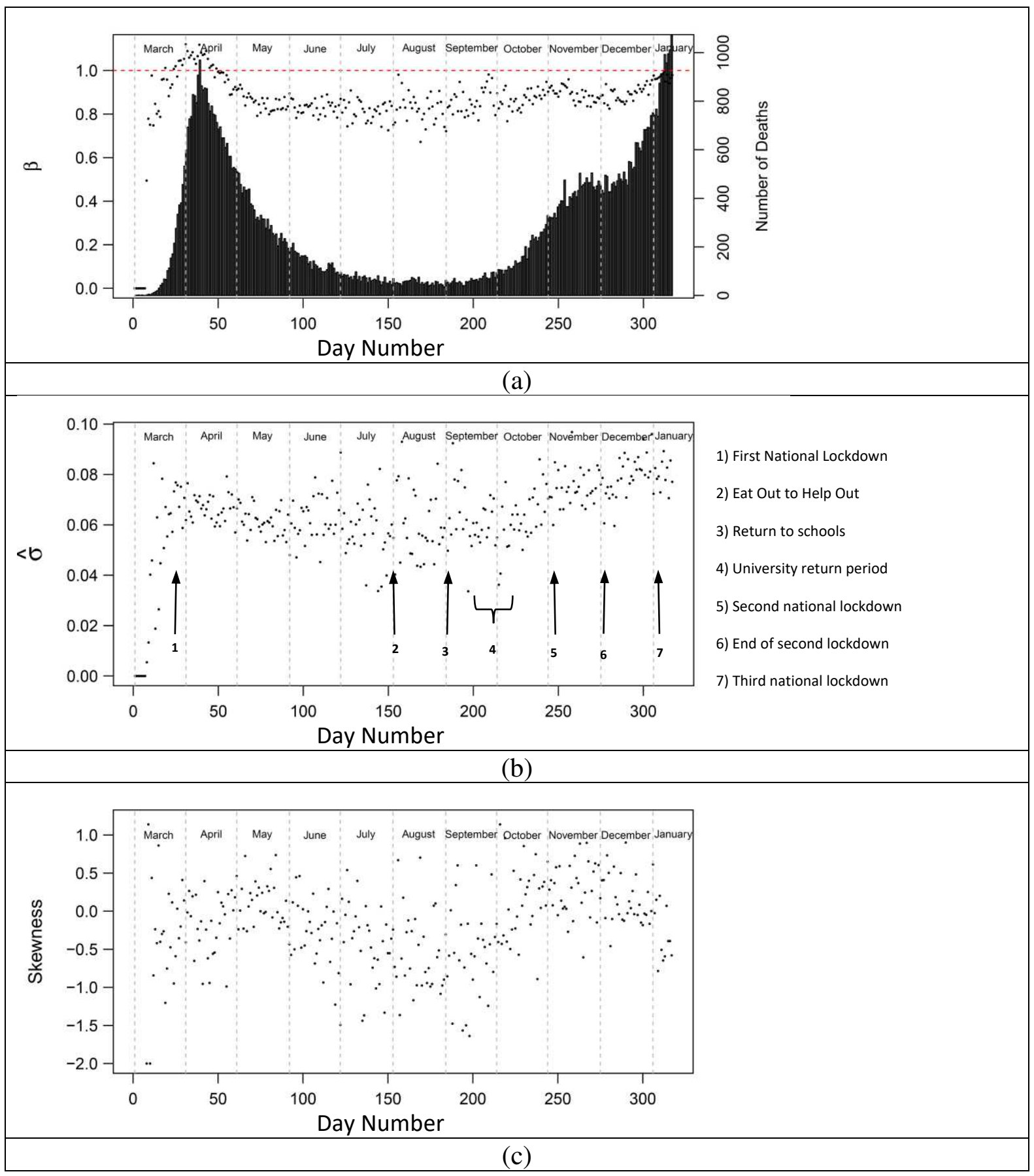

Fig 5. Daily time series of scaling exponent and residual variance and skewness for deaths 01/03/2020 and 11/01/2021. (a) Time series of daily scaling exponent of COVID-19 deaths, (b) residual variance, and (c) residual skewness. The horizontal line in (a) indicates linear scaling. The bar chart indicates raw daily deaths. Arrows in (b) indicate key dates/time periods. The second lockdown in Wales preceded England beginning on 20/10/2020.

Dispersion of COVID-19 Case residuals over time. To better understand the distribution of residuals, we investigate the normal and generalized logistic distributions as candidate 
distributions using the LTLA data (Equation 5). The normal distribution is symmetric and has no skew. The GL distribution has three parameters which can accommodate a wider range of shapes including positive and negative skewing. When comparing normal and GL distributions as models for scaling law residuals the additional parameter needs to be accounted for. We used the Akaike (AIC) and Bayesian (BIC) information criteria to decide if normal or GL represented a better model for each day in the 10-month period. When selecting a model, lower AIC and BIC scores represent better fits. The differences between AIC and BIC scores obtained from fitting the two distributions to the residuals were for each day in the 10-month period (Fig. 6). Positive values correspond to a generalised logistic distribution as the preferred model, whilst a negative value corresponds to a normal distribution as the preferred model. All daily histograms for cases and deaths can be found in Figures S7 and S8 in the supplementary material.

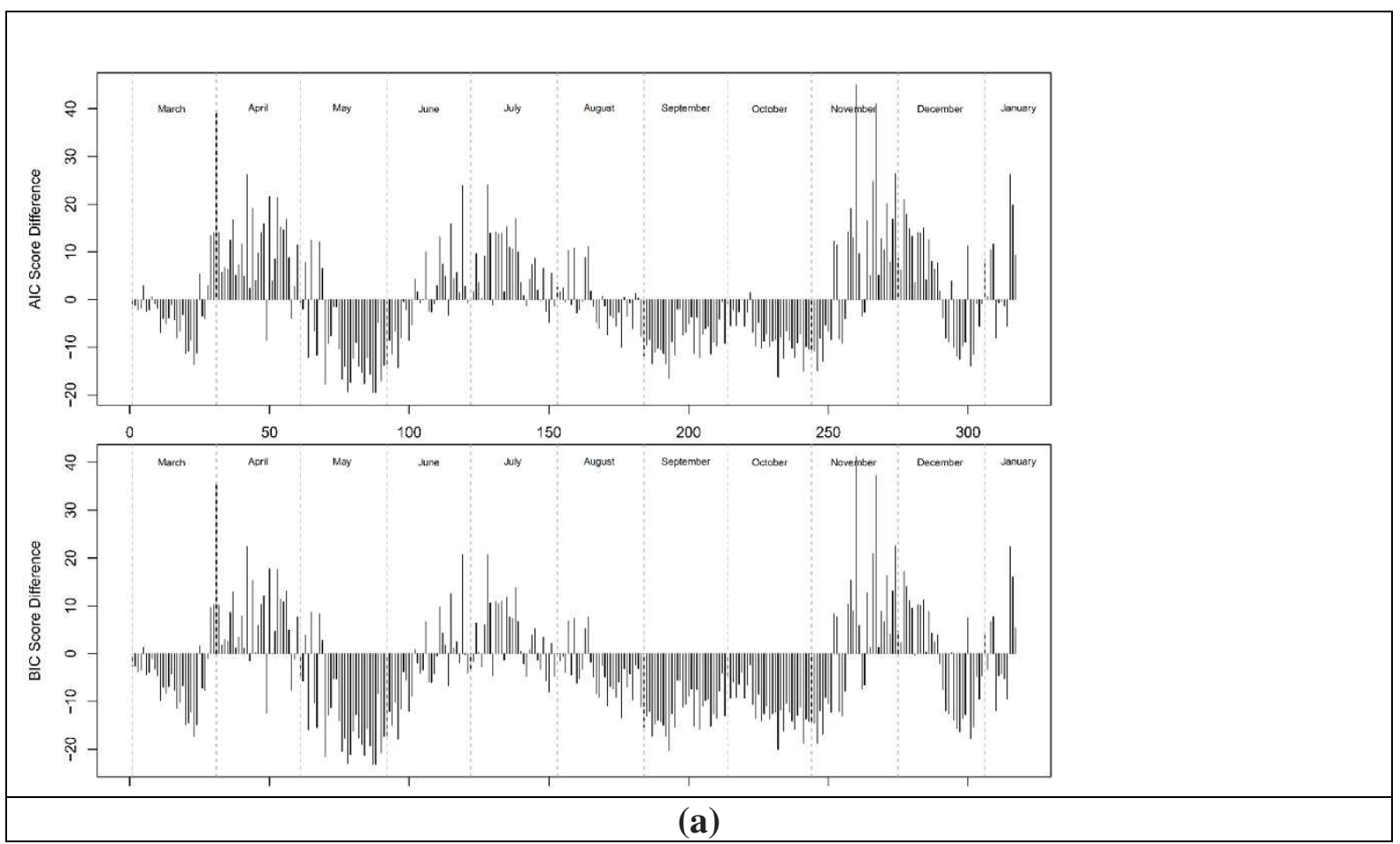




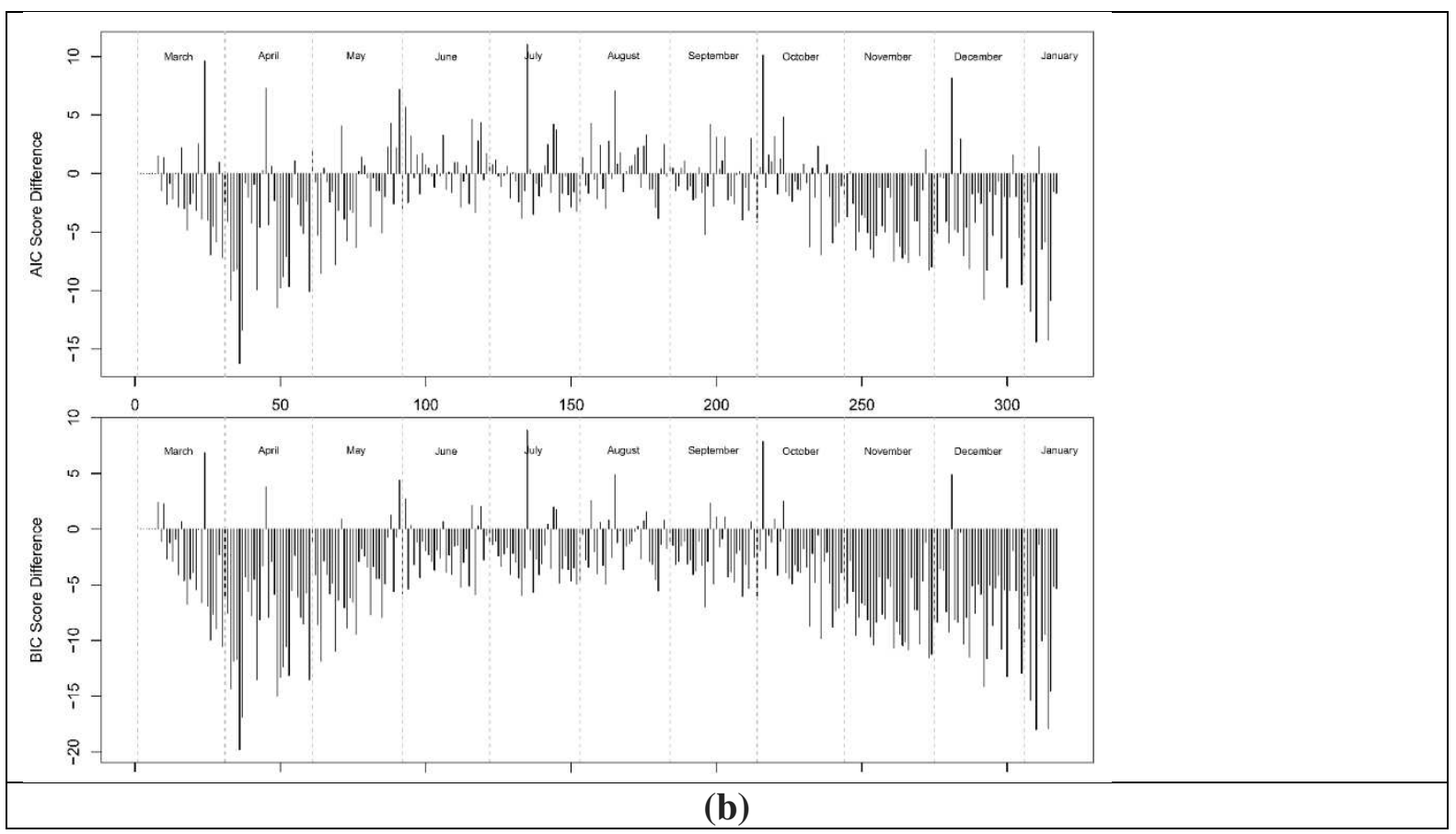

Fig 6. AIC and BIC differences over time. (a) COVID-19 cases and (b) COVID-19 death. Positive AIC/BIC indicates GL is a better fit and a negative AIC/BIC indicates normal is a better fit.

Although there is some noise in the differences, the contrast between cases and death is again clear. During the initial periods of the lockdowns (March, November and January) propagation of cases was associated with a GL distribution and negative skew whilst during less restrictive time frames (August, September, October) propagation is associated with a normal distribution. Modelling and simulation of propagation [40] using network science and a gamma distribution have been attempted using varying parameter values to represent different proportions of "super-spreaders." This analysis indicated that the initial trajectory of exposed and infected people in a population accelerates quickly in networks where there are a high proportion of super-spreaders. Our analysis makes clear a gamma distribution cannot accommodate the range of shapes required over the full period of a pandemic. A gamma distribution cannot be negatively skewed and the structure of cases in the pandemic has periods of negative skewing. "Super-spreading" is almost certainly important but understanding the converse concepts of "super-isolation" and cold-spots which can better concepts for defining the features of a 
distribution need to be appreciated. It is also useful to better understand the characteristics and features of regions where a disease is not spreading or is consistently below expectation. The contrasting behaviour of deaths is also of interest. Normally distributed residuals were the overwhelming feature of the 10 month period.

\section{Conclusions}

This study has established that both regional per capita measures and scaling law residuals exhibit both positive and negative skewing. Positively skewed distributions have been used to model pandemic behaviour [40]. This is important to indicate super-spreading and hot-spots, but insufficient to characterise the full sweep of a pandemic.

Similarly, scaling law parameters are often thought to be constant or very slowly changing features of a process. In the case of COVID-19 cases, scaling parameters evolved over relatively short periods of time. For cases, the scaling law exponents reached a peak at the beginning of the first lockdown and gradually declined for approximately three months. Preferential propagation of COVID-19 cases switched between rural and urban regions multiple times. COVID-19 mortality gave a more consistent picture of low population density regions preferentially and consistently affected with linear scaling only being approached at the beginning and end of the 10-month period.

Variance is a key descriptor of the distribution of regional cases. Lockdowns produce heterogeneity (higher variance) across regions while reducing cases. The re-opening of schools drove heterogeneity during a period of case growth indicative of locally important outbreaks. Country scale mixing such as occurred with the opening of universities and holiday periods promotes homogenisation (low variance). All key statistical metrics from regional death data were remarkably different from cases in the time period. This is consistent with regional age demographics in England and Wales. From a policy point of view these observations and 
patterns are particularly important, as they provide insight and expected indicative effects following implementation of health policies.

Within this framework it is important to note that England and Wales had continuous community spread of SARS-COV-2. Excepting the very early period in March, there has been nothing that could be called a "surge." Within the 10-month period, the rise and fall of cases and deaths have been gradual as has the evolution of scaling metrics, variance structures, and distribution shapes.

\section{Materials and Methods:}

Data Sets. England and Welsh data on the number of daily COVID-19 cases and deaths were obtained from Public Health England (PHE). (https://coronavirus.data.gov.uk/) for lower tier local authorities (LTLAs). England and Wales population estimates were based on the 2011 census and regional land areas were obtained from NOMIS (https://www.nomisweb.co.uk), a database service run by the University of Durham on behalf of the UK Office for National Statistics. The shape files for LTLAs were obtained from the open geography portal (http://geoportal.statistics.gov.uk) provided by the UK Office for National Statistics and UK Data Service (https://census.ukdataservice.ac.uk). English and Welsh LTLAs for COVID-19 cases, COVID-19 mortality, population, and area were aligned in a daily time series covering the period from $01 / 03 / 2020$ to $11 / 01 / 2021$. All data in this study are publicly available under Crown Copyright. The data are provided by PHE such that counts between 0 and 2 are blank in all CSV files and NULL in any other formats. These adaptations in the data are to prevent disclosure control issues. In the analysis, zero values representing null returns were considered as missing values. The population in LTLA data for City of London and Isles of Scilly are considered small and therefore statistics in these regions were combined with Hackney and Cornwall respectively. 
Statistical Analysis. The data were analysed using the statistical software R version (3.6.2) [19] with the sf (0.9-1) [20], raster (3.0-12) [21], dplyr (0.8.5) [22], spData (0.3.5) [23], tmap (2.32) [24], ggplot2 (3.3.0) [25-28], xlsx (0.5.7) [29], gplots (3.0.4) [30], httr (1.4.2) [31], plyr (1.8.5) [32], png (0.1-7) [33], rgdal (1.5-19) [34], rgeos (0.5-5) [35], lubridate (1.7.9.2) [36], fitdistrplus (1.1-3) [37], fgarch (3042.83.2) [38] and glogis (1.0-1) [39] packages.

\section{Data Availability}

All data generated or analysed during this study are included in this published article (and its supplementary information files). This data was compiled from a range of publicly available sources as noted in the manuscript. These are provided as the following files: UK_regions.xlsx, UK_daily_cases.xlsx, UK_daily_death.xlsx and UK_daily_total.xlsx.

\section{Code Availability}

We have also provided a R-script as supplementary information. This has been provided as code_version2.R.

\section{Acknowledgements}

The authors are grateful to the UK Office of National Statistics and Public Health England for making these data available.

\section{References}

1. L. Morawska \& J. Cao, Airborne transmission of SARS-CoV-2: The world should face the reality. Environment International, 139 (2020) 105730. https://doi.org/10.1016/j.envint.2020.105730.

2. E. L. Anderson, P. Turnham, J. R. Griffin, \& C. C. Clarke, Consideration of the Aerosol Transmission for COVID-19 and Public Health. Risk Analysis, (2020). https://doi.org/10.1111/risa.13500.

3. K. A. Prather, C. C. Wang, \& R. T. Schooley, Reducing transmission of SARS-CoV-2. Science, 368 (2020) 1422-1424. https://doi.org/10.1126/science.abc6197.

4. S. Asadi, N. Bouvier, A. S. Wexler, \& W. D. Ristenpart, The coronavirus pandemic and aerosols: Does COVID-19 transmit via expiratory particles? Aerosol Science and Technology, 54 (2020) 635-638. https://doi.org/10.1080/02786826.2020.1749229. 
5. M. Schläpfer, L. M. A. Bettencourt, S. Grauwin, M. Raschke, R. Claxton, Z. Smoreda, G. B. West, \& C. Ratti, The scaling of human interactions with city size. Journal of The Royal Society Interface, 11 (2014) 20130789. https://doi.org/10.1098/rsif.2013.0789.

6. A. J. Stier, M. G. Berman, \& L. M. A. Bettencourt, COVID-19 attack rate increases with city size. medRxiv, (2020) 1-23. https://doi.org/10.1101/2020.03.22.20041004.

7. A. Ascani, A. Faggian, \& S. Montresor, The geography of COVID-19 and the structure of local economies: The case of Italy. Journal of Regional Science, (2020) 135. https://doi.org/10.1111/jors.12510.

8. B.-H. F. Cardoso \& S. Gonçalves, Urban Scaling of COVID-19 epidemics. arXiv preprint arXiv:2005.07791, (2020).

9. H. V. Ribeiro, A. S. Sunahara, J. Sutton, M. Perc, \& Q. S. Hanley, City size and the spreading of COVID-19 in Brazil. PLOS ONE, 15 (2020) e0239699. https://doi.org/10.1371/journal.pone.0239699.

10. H. V. Ribeiro, D. Rybski, \& J. P. Kropp, Effects of changing population or density on urban carbon dioxide emissions. Nature Communications, 10 (2019) 3204. https://doi.org/10.1038/s41467-019-11184-y.

11. J. Sutton, G. Shahtahmassebi, H. V. Ribeiro, \& Q. S. Hanley, Rural-urban scaling of age, mortality, crime and property reveals a loss of expected self-similar behaviour. Scientific Reports, 10 (2020) 16863. https://doi.org/10.1038/s41598-020-74015-x.

12. H. V. Ribeiro, Q. S. Hanley, \& D. Lewis, Unveiling relationships between crime and property in England and Wales via density scale-adjusted metrics and network tools. PLOS ONE, 13 (2018) e0192931. https://doi.org/10.1371/journal.pone.0192931.

13. Q. S. Hanley, D. Lewis, \& H. V. Ribeiro, Rural to Urban Population Density Scaling of Crime and Property Transactions in English and Welsh Parliamentary Constituencies. PLOS ONE, 11 (2016) e0149546. https://doi.org/10.1371/journal.pone.0149546.

14. L. M. A. Bettencourt, J. Lobo, D. Helbing, C. Kuhnert, \& G. B. West, Growth, innovation, scaling, and the pace of life in cities. Proceedings of the National Academy of Sciences, 104 (2007) 7301-7306. https://doi.org/10.1073/pnas.0610172104.

15. E. Bokányi, Z. Szállási, \& G. Vattay, Universal scaling laws in metro area election results. PLOS ONE, 13 (2018) e0192913. https://doi.org/10.1371/journal.pone.0192913.

16. L. G. A. Alves, H. V. Ribeiro, E. K. Lenzi, \& R. S. Mendes, Distance to the Scaling Law: A Useful Approach for Unveiling Relationships between Crime and Urban Metrics. PLoS ONE, 8 (2013) e69580. https://doi.org/10.1371/journal.pone.0069580.

17. P. H. England, Coronavirus (COVID-19) in the UK. (n.d.). https://coronavirus.data.gov.uk/ (accessed February 23, 2021).

18. L. M. A. Bettencourt, J. Lobo, D. Strumsky, \& G. B. West, Urban Scaling and Its Deviations: Revealing the Structure of Wealth, Innovation and Crime across Cities. PLoS ONE, 5 (2010) e13541. https://doi.org/10.1371/journal.pone.0013541.

19. R, The R Project for Statistical Computing. (n.d.). https://www.r-project.org/ (accessed 
February 23, 2021).

20. E. Pebesma, Simple Features for R: Standardized Support for Spatial Vector Data. The R Journal, 10 (2018) 439. https://doi.org/10.32614/RJ-2018-009.

21. R. J. Hijmans, raster: Geographic Data Analysis and Modeling. (2020).

22. H. Wickham, R. Francois, L. Henry, \& K. Muller, dplyr: A Grammar of Data Manipulation. (2019).

23. R. Bivand, J. Nowosad, \& R. Lovelace, spData: Datasets for Spatial Analysis. (2020).

24. M. Tennekes, tmap : Thematic Maps in R. Journal of Statistical Software, 84 (2018) 1-39. https://doi.org/10.18637/jss.v084.i06.

25. R. Lovelace \& J. Cheshire, Introduction to visualising spatial data in R (2017). https://doi.org/10.5281/zenodo.889551.

26. R. Lovelace \& J. Cheshire, Introduction to visualising spatial data in R Part I: Introduction. Tutorial, (2015). https://doi.org/10.5281/zenodo.889551.

27. J. Cheshire \& R. Lovelace, Introduction to Spatial Data and ggplot2. RPub, (2013).

28. H. Wickham, ggplot2: Elegant Graphics for Data Analysis (Springer-Verlag New York, 2016).

29. A. A. Dragulescu \& C. Arendt, xlsx: Read, Write, Format Excel 2007 and Excel 97/2000/XP/2003 Files. (2018).

30. G. R. Warnes, B. Bolker, L. Bonebakker, R. Gentleman, W. Huber, A. Liaw, T. Lumley, M. Maechler, A. Magnusson, S. Moeller, M. Schwartz, \& B. Venables, gplots: Various R Programming Tools for Plotting Data. (2020).

31. H. Wickham, httr: Tools for Working with URLs and HTTP. (2020).

32. H. Wickham, The Split-Apply-Combine Strategy for Data Analysis. Journal of Statistical Software, 40 (2011) 1-29.

33. S. Urbanek, png: Read and write PNG images. (2013).

34. R. Bivand, T. Keitt, \& B. Rowlingson, rgdal: Bindings for the "Geospatial" Data Abstraction Library. (2021).

35. R. Bivand \& C. Rundel, rgeos: Interface to Geometry Engine - Open Source ('GEOS'). (2020).

36. G. Grolemund \& H. Wickham, Dates and Times Made Easy with Lubridate. Journal of Statistical Software, 40 (2011) 1-25.

37. M. L. Delignette-Muller \& C. Dutang, An $\{$ R $\}$ Package for Fitting Distributions. Journal of Statistical Software, 64 (2015) 1-34.

38. D. Wuertz, T. Setz, Y. Chalabi, C. Boudt, P. Chausse, \& M. Miklovac, Rmetrics Autoregressive Conditional Heteroskedastic Modelling. (2020).

39. A. Zeileis \& T. Windberger, Fitting and Testing Generalized Logistic Distributions. (2018).

40. O. Reich, G. Shalev, \& T. Kalvari, Modeling COVID-19 on a network: super- 
spreaders, testing and containment. medRxiv 2020.04.30.20081828, (2020). https://doi.org/10.1101/2020.04.30.20081828. 
Figures

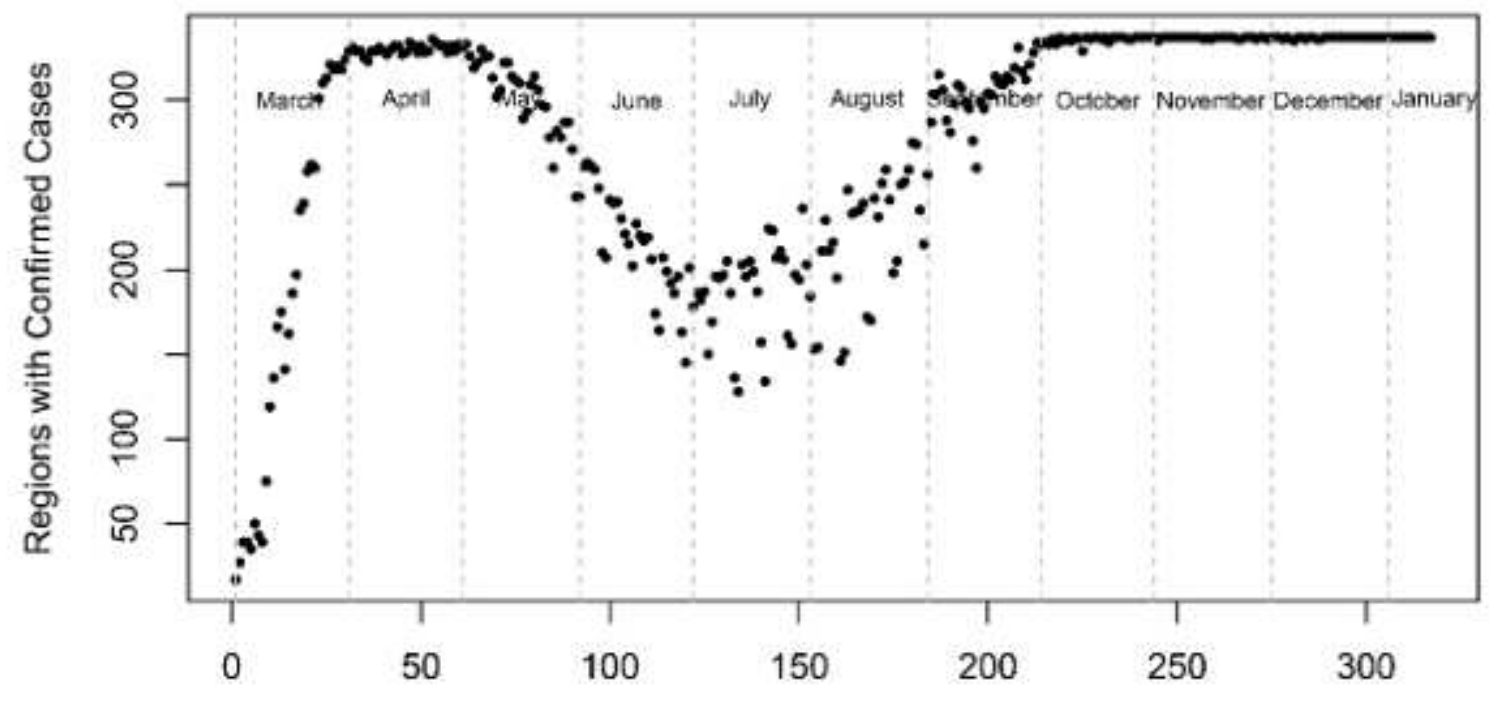

Day Number

(a)

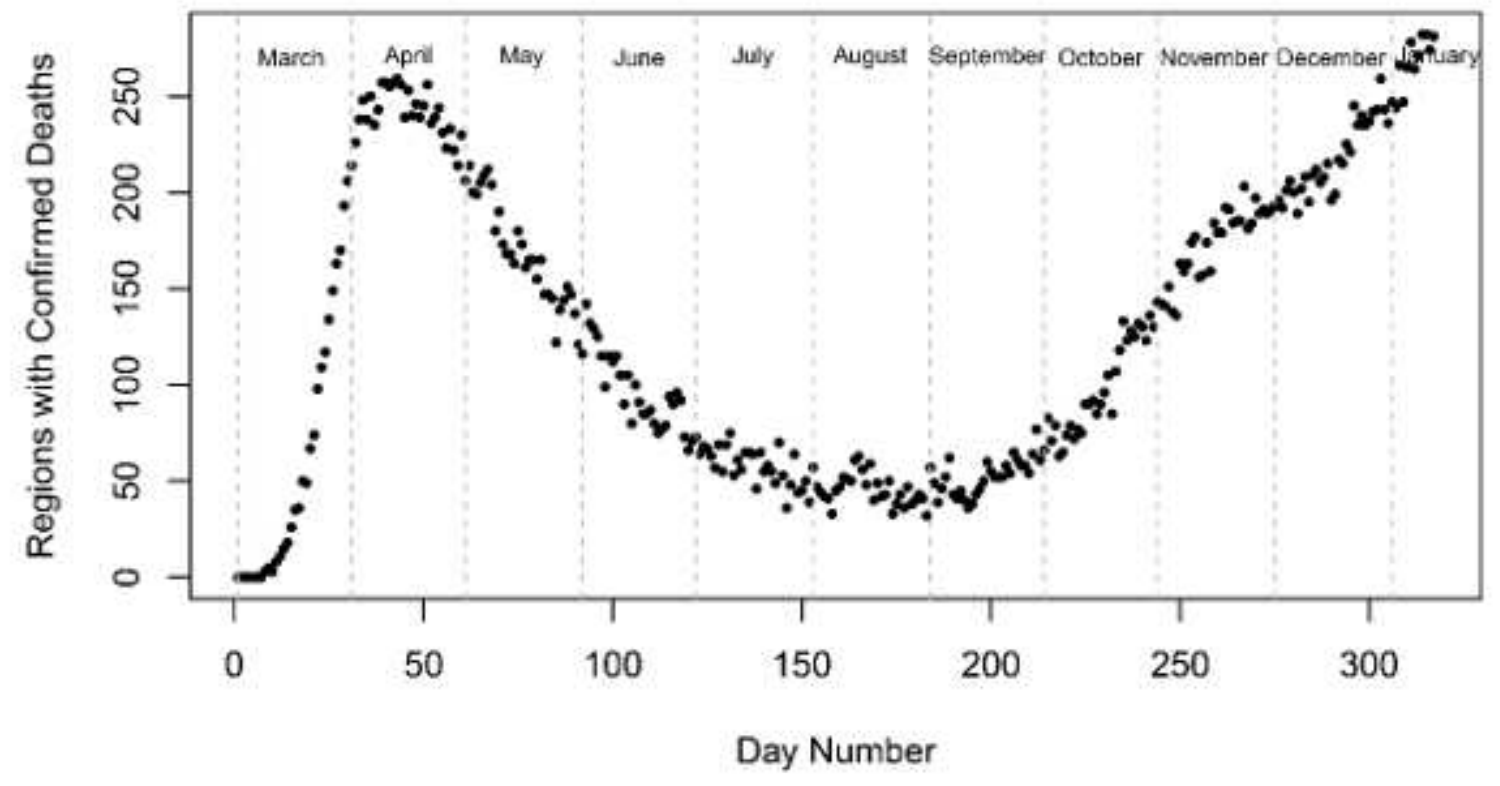

(b)

Figure 1

Time series indicating the number of LTLAs returning cases (a) or deaths (b) over the period of study. 


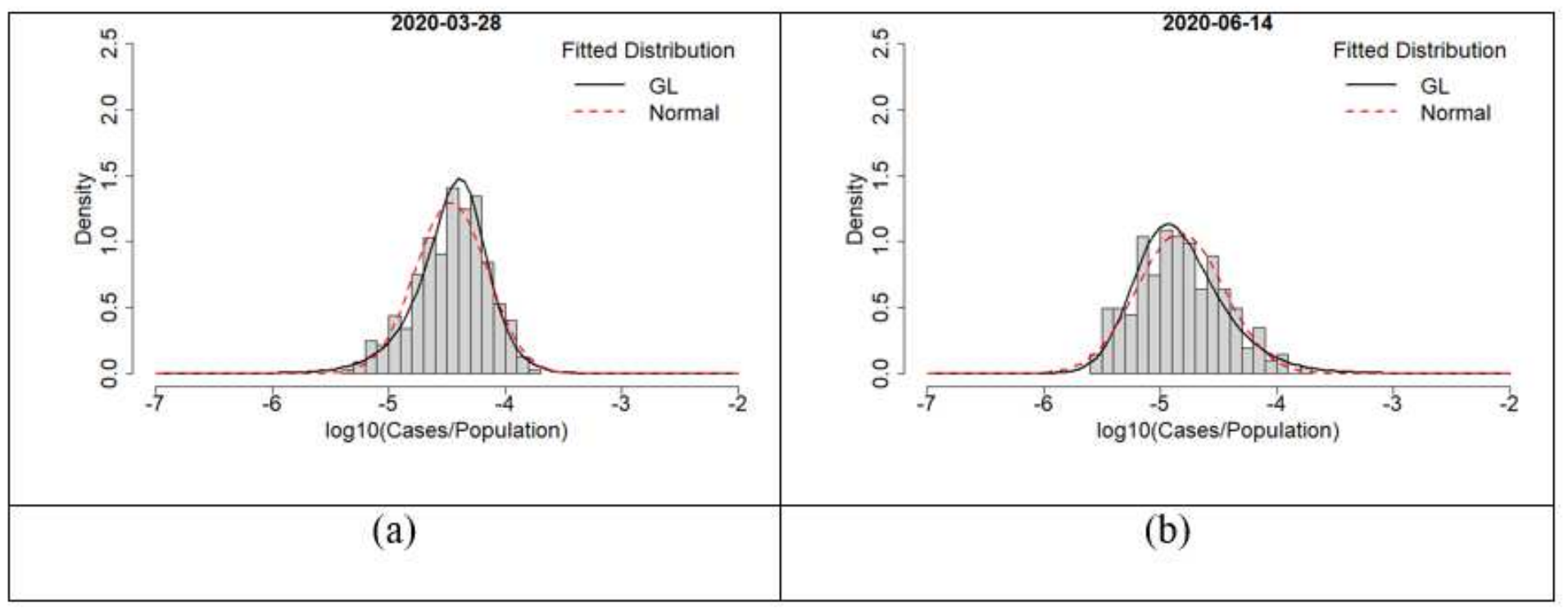

\section{Figure 2}

Histograms of per capita cases in English and Welsh LTLAs. Some periods within the time series showed negative skew (a) while others were positively skewed (b).

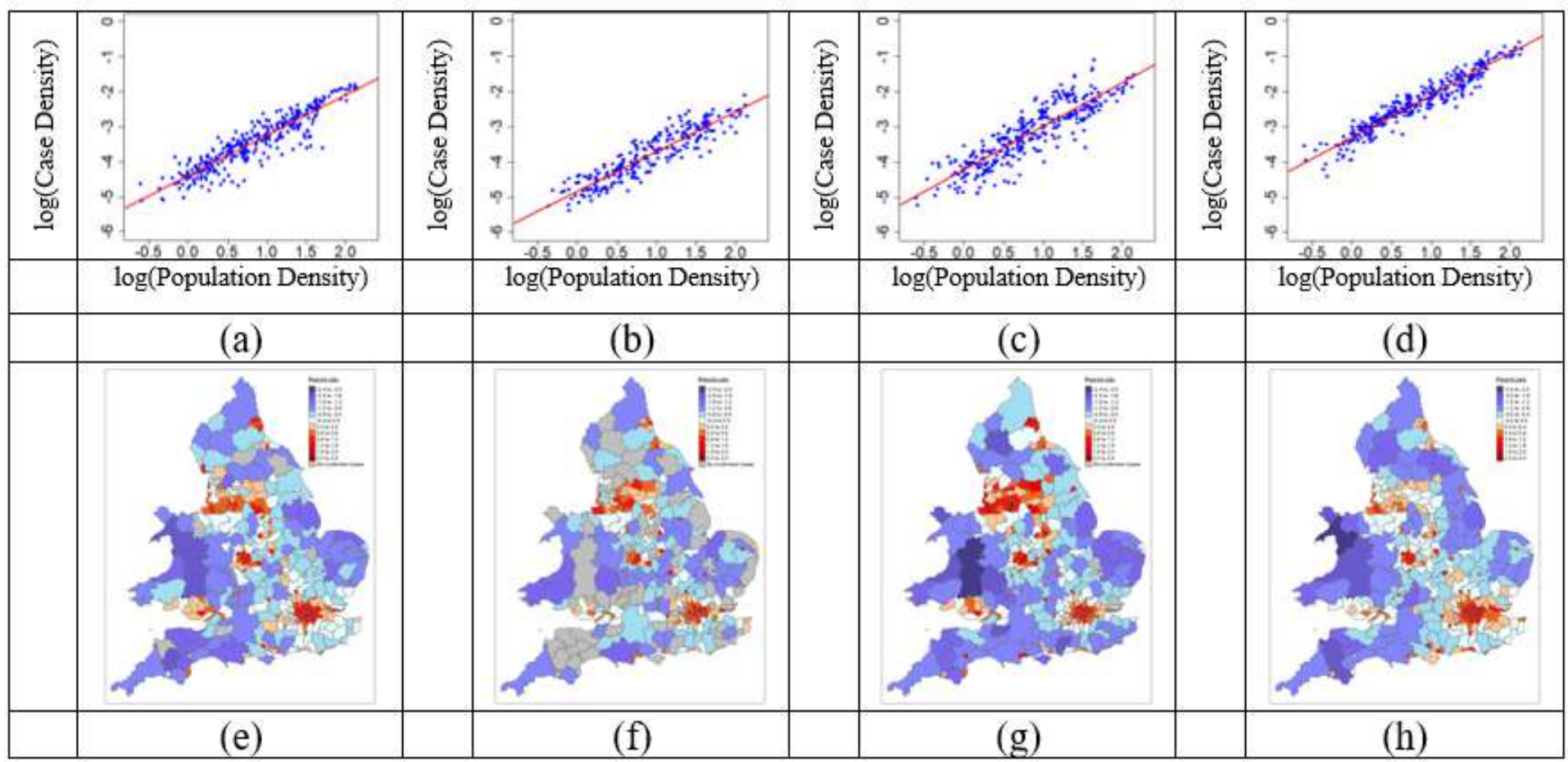

\section{Figure 3}

Scaling plots and geoplots at different times during the pandemic. These are recorded on the (a and e) 31/03/2020. (b and f) 31/8/2020. ( $\mathrm{c}$ and g) 30/09/2020. ( $\mathrm{d}$ and h) 31/12/2020. Regions that are red are above expectation and blue is below. The darker the shade the further from the scaling law. 


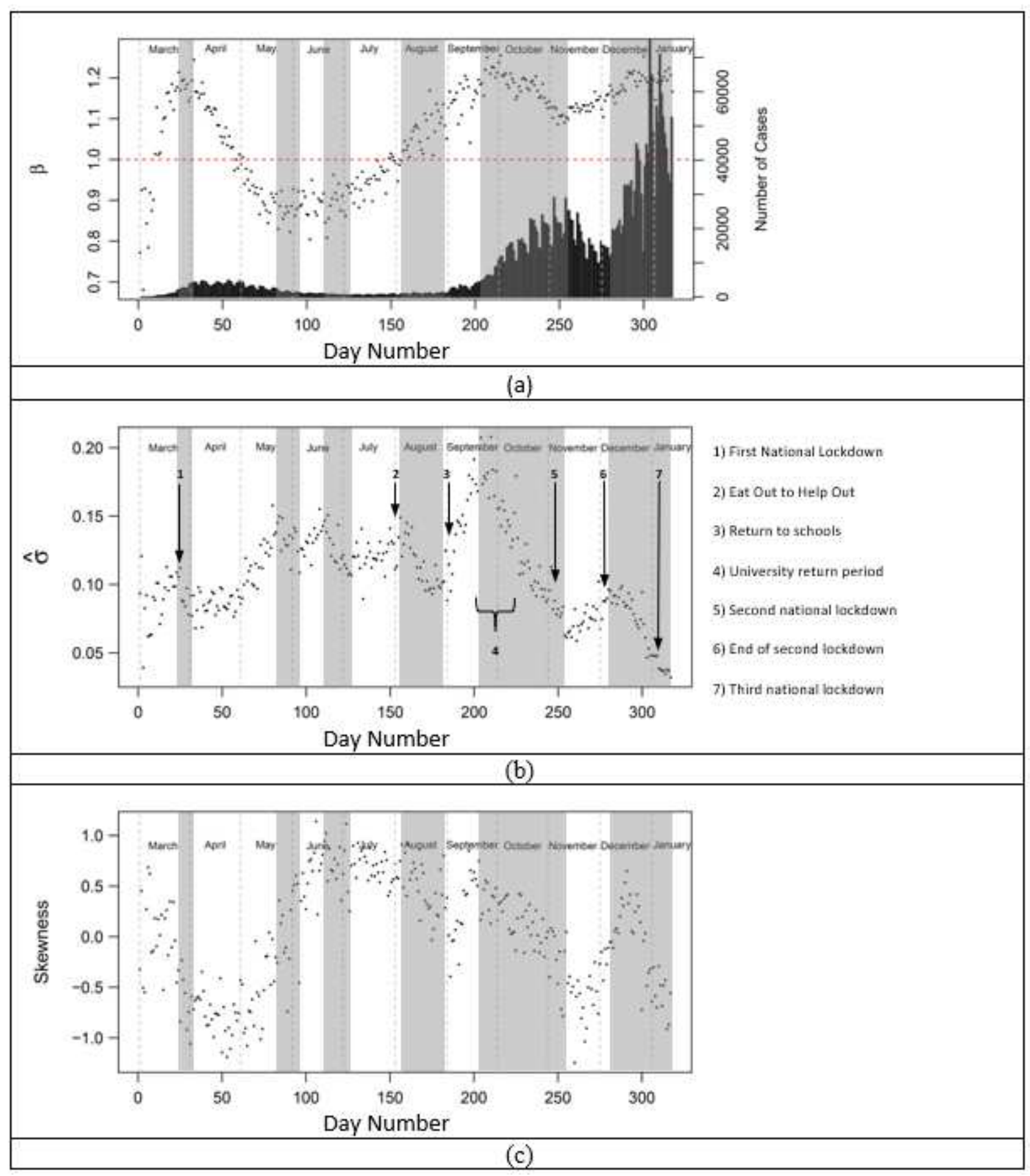

\section{Figure 4}

Daily time series of scaling exponent and residual variance and skewness for cases between 01/03/2020 and 11/01/2021. (a) Time series of daily scaling exponent of COVID-19 cases, (b) residual variance, and (c) residual skewness. The horizontal line in (a) indicates linear scaling. The bar chart indicates raw daily cases. The grey shading indicates periods of homogenisation. Arrows indicate key dates/time periods. The second lockdown in Wales preceded England beginning on 20/10/2020. 


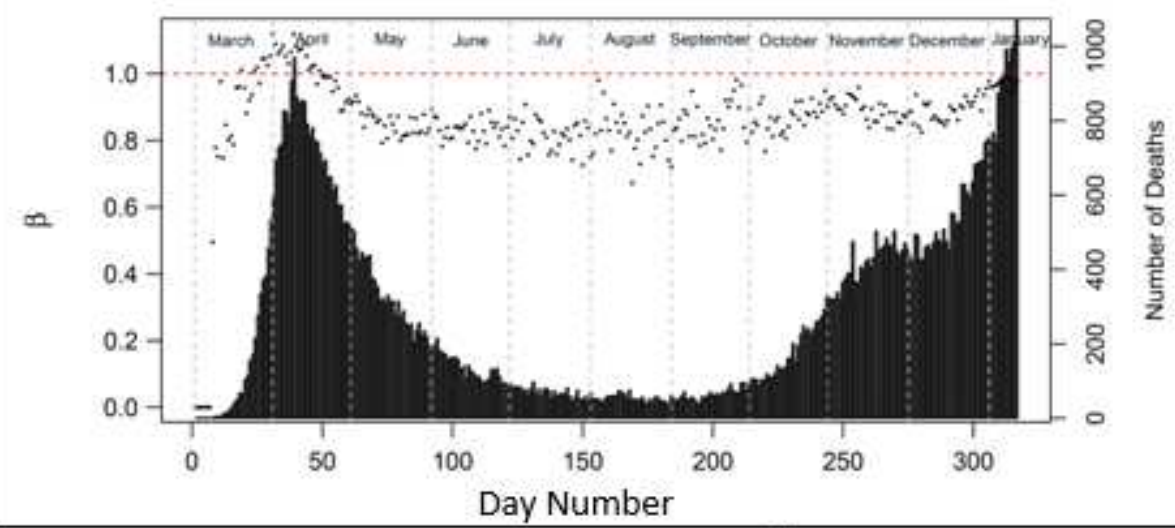

(a)

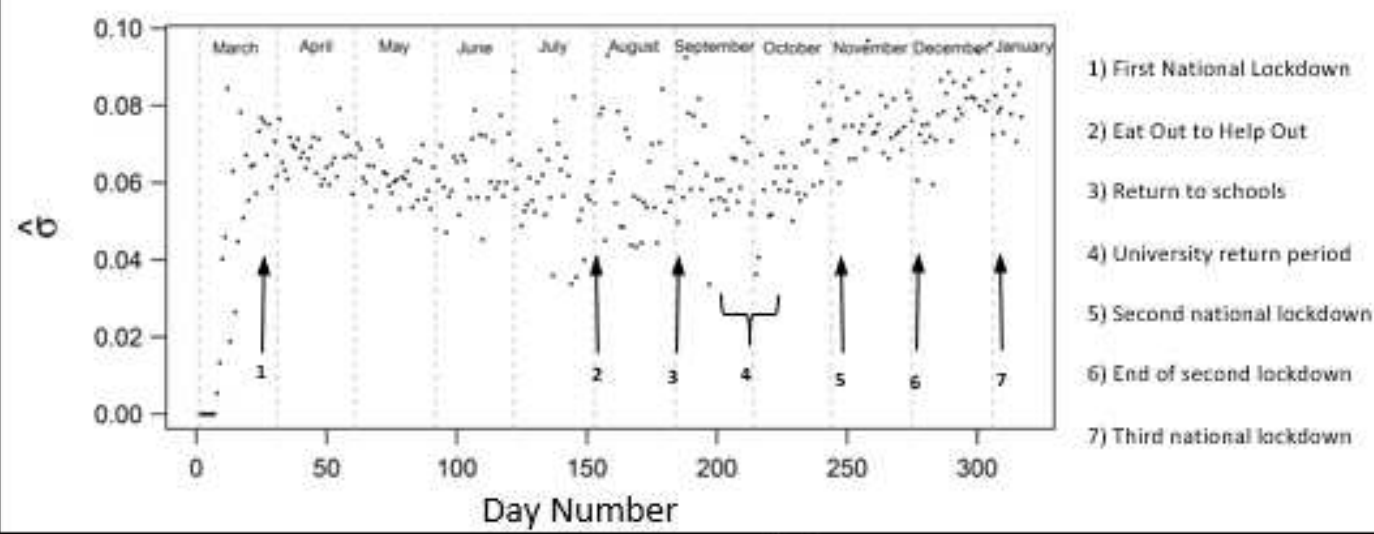

(b)

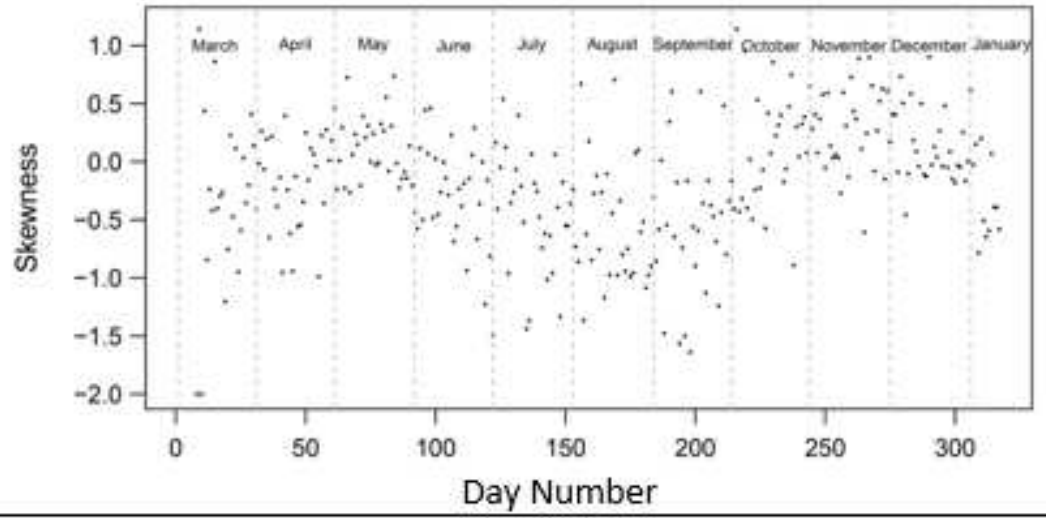

(c)

\section{Figure 5}

Daily time series of scaling exponent and residual variance and skewness for deaths 01/03/2020 and 11/01/2021. (a) Time series of daily scaling exponent of COVID-19 deaths, (b) residual variance, and (c) residual skewness. The horizontal line in (a) indicates linear scaling. The bar chart indicates raw daily deaths. Arrows in (b) indicate key dates/time periods. The second lockdown in Wales preceded England beginning on 20/10/2020. 


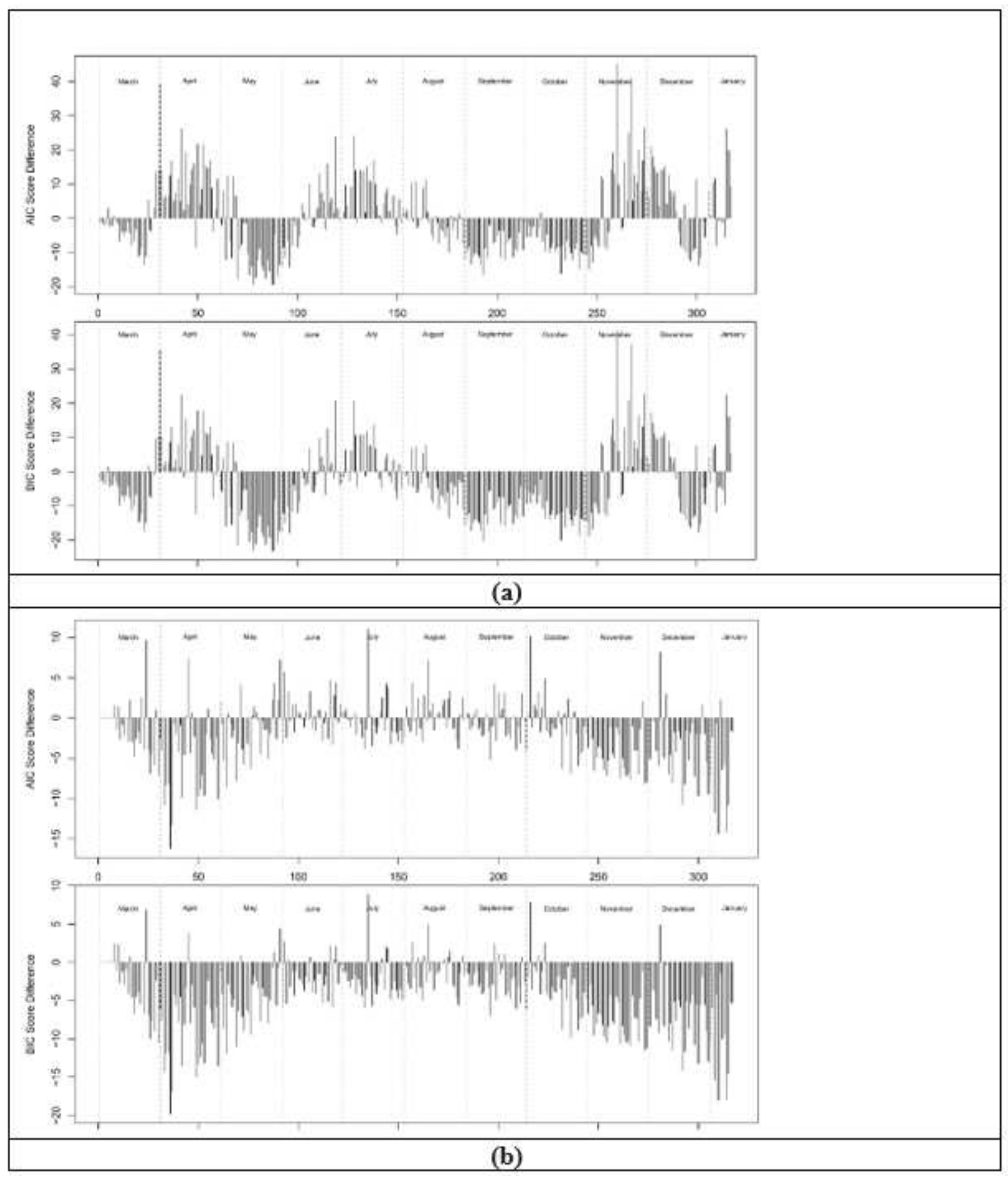

Figure 6

AIC and BIC differences over time. (a) COVID-19 cases and (b) COVID-19 death. Positive AIC/BIC indicates $\mathrm{GL}$ is a better fit and a negative AIC/BIC indicates normal is a better fit.

\section{Supplementary Files}

This is a list of supplementary files associated with this preprint. Click to download. 
- HomogeneousandheterogeneouspropagationofCOVIDsupplement.docx

- codeversion2.r

- UKregions.xlsx

- UKdailydeaths.xIsx

- UKdailytotal.xlsx

- UKdailycases.xlsx 